\title{
ESTRUCTURAS AFECTIVAS TRIANGULARES Y PRODUCCIÓN DE DESEO EN LA FILOSOFÍA DE SPINOZA
}

\author{
TRIANGULAR AFFECTIVE STRUCTURES AND PRODUCTION OF DESIRE IN THE \\ PHILOSOPHY OF SPINOZA
}

\author{
Mario Donoso Gómez* \\ Université Paris 8, \\ Paris - Francia. \\ Recibido enero de 2018/Received January, 2018 \\ Aceptado abril de 2018/Accepted April, 2018

\section{RESUMEN}

Este artículo analiza un conjunto de estructuras afectivas en la filosofía espinosista cuya principal característica es que no son binarias, no se reducen a la fórmula sujeto-objeto, sino que implican un tercero. Así, distinguiremos dos grupos, el primero, en el que uno de los polos es un objeto mientras que los otros dos son humanos, y el segundo, en el que los tres polos son hombres. Esta distinción nos permitirá analizar el proceso de formación de las relaciones interhumanas.

Palabras Clave: otro, Otro, imitación, rivalidad, propiedad, indignación, deseo.

\begin{abstract}
This article analyzes a set of affective structures in Spinoza's philosophy whose main characteristic is that are not binary; they cannot be reduced to the formula subject-object, imply a third element. Thus, two groups will be distinguished: the first, in which one of the poles is an object while other two are human, and the second, in which the three poles are men. This distinction will allow us to analyses the formation process of forming inter-human relations.
\end{abstract}

Key Words: other, Other, imitation, rivalry, property, indignation, desire.

\section{Introducción}

Después de haber definido la esencia del individuo como conato <conatus $>$ ( $E$ III 7; Spinoza, 2000, p. 133) $)^{1}$ y de explicar que el hombre tiene conciencia de ese esfuerzo $(E$ III 9; Spinoza, 2000, p. 133) $)^{2}$, Spinoza, en el escolio de la proposición 9 de la tercera parte de la Ética, identifica el conato con el deseo $<$ cupiditas $>$, que no es sino la búsqueda consciente, por parte del hombre, de aquello que contribuye a su conservación ( $E$ III 9 sc.; Spinoza, 2000, p. 134) $)^{3}$. Sin embargo, esta definición abstracta no permite ver toda la complejidad del asunto, pues los hombres desean cosas muy dispares, muchas de las cuales son poco convenientes a su conservación, ya sea

* Autor correspondiente / Correspondig author: mdonosogom@gmail.com 
porque no siempre desean lo que más conviene a su naturaleza o porque prefieren las cosas que les producen un gozo más inmediato, aunque este sea un gozo desequilibrado o mutilado $<$ titilatio $>$. Por esta razón, para saber en qué consiste el deseo de los hombres no basta con enunciar su funcionamiento abstracto sino que es necesario analizar los mecanismos a partir de los cuales los hombres desean cosas particulares.

La mayor parte de los deseos concretos de los hombres se explican por la representación que los hombres tienen de las cosas. El origen de la representación es la manera en la que las cosas nos afectan; así, cuando una cosa nos afecta de manera gozosa, es decir, cuando aumenta nuestro Gozo $<$ Laetitia $>$, nos representamos esta cosa de manera deseable. Para Spinoza, representar una cosa de manera deseable y desearla es lo mismo; hay, por lo tanto, una identificación entre la representación de una cosa gozosa y el deseo. Sin embargo ésta no es la única manera de adquirir un objeto de deseo. En la tercera parte de la Ética, partiendo del desarrollo de los afectos interhumanos, Spinoza va a elaborar explícita o implícitamente otras formas de desear en relación con los otros cuya base es la imitación de los afectos <imitatio affectuum $>$. Este mecanismo imitativo, que aparece en la proposición 27 (E III 27; Spinoza, 2000, p. 144), permite pensar un tipo de deseo que no proviene de la manera en la que una cosa nos afecta sino de la manera en que afecta a los demás: por la imitación nos apropiamos de los afectos ajenos, entre ellos del deseo; esta apropiación del deseo es llamada emulación <aemulatio> por Spinoza (E III 27 sc.; Spinoza 2000, p. 144). Sin embargo, no es esta la única forma de deseo que conlleva la imitación. La imitación implica una especie de deseo cuyo objeto son los otros, que puede traducirse en un deseo de sociabilidad y en un deseo de dominación.

La socialización es un mecanismo complejo que parte de una contradicción: los hombres quieren que los demás sean como ellos y para eso lo que hacen es asemejarse a los demás. Esta contradicción de la sociabilidad primaria puede encontrarse tanto en afectos como la ambición como en mecanismos como la imitación. Alexandre Matheron distingue dos tipos de ambición en la teoría afectiva espinosista. La primera es la ambición de gloria, que consiste en hacer lo que place al otro para que el otro nos ame. Los hombres, de manera egoísta, quieren ser amados por los demás, pero para ello tienen que comportarse como si fueran altruistas, tienen que hacer lo que place a los demás. Por esta razón, como Matheron señala, la ambición permite tejer una sociabilidad básica a partir del "lugar original donde el egoísmo y el "altruismo" coinciden" (Matheron, 1969, p. 164). Sin embargo hay otro tipo de ambición que Matheron llama ambición de dominación (Matheron, 1969, p. 168), que en el fondo es un deseo de someter y tiranizar a los demás. En oposición a la ambición de gloria, este tipo de ambición acelera la desintegración social y el provoca el conflicto.

Para Pierre-François Moreau esta contradicción de hacer lo que los otros desean movidos por el deseo de que los otros hagan lo que queremos se encuentra en la imitación afectiva. Según Moreau, la imitación afectiva es una especie de sumisión indirecta que consiste en imitar al otro para que este nos imite. Queremos que el otro sea como nosotros y para ello queremos que nos imite, pero la única manera de hacerlo es imitándole:

Los mecanismos de esta última explican por qué, estando inclinados a imponer al prójimo el ingenium propio, los hombres son al mismo tiempo llevados a identificarse, al menos por un cierto tiempo y en cierta medida, con el ingenium de otro. (Moreau, 2009, p. 6).

Sin embargo, esta socialización básica no impide la aparición de otros conflictos mayores que provienen del deseo desmesurado de los individuos de someter a los demás para concebirse superiores a ellos. La estabilidad afectiva no suprime lo que Matheron llama ambición de dominación (Matheron, 1969, p. 168) o Macherey denomina la dimensión tiránica (Macherey, 1995, p. 253), que es el deseo de todo hombre de someter a los demás para que sean como él. Es en este contexto donde aparece la nueva estructura triangular en la que las partes están formadas por formas subjetivas.

La manera en la que estos dos mecanismos aparecen en el texto parece seguir una lógica binaria: hay un individuo que quiere someter a otro. En cambio, para comprender estos mecanismos, es necesario, a nuestro juicio, introducir un tercer elemento. Del mismo modo que la imitación modifica la relación de posesión del objeto al introducir un tipo de representación y de deseo que no proviene de la afectación directa del objeto, la imitación introduce de manera implícita un tercer elemento 
en las relaciones interhumanas. El objetivo de este artículo es evidenciar este tercer elemento y mostrar su articulación para comprender mejor los mecanismos intersubjetivos que forman los lazos entre los hombres. Para llevar a cabo este análisis distinguiremos dos partes: la primera, estudiará por un lado la estructura triangular paralela a la imitación-ambición, o socialización primaria; la segunda, analizará la estructura de la dominación, en la que se encuentra a su vez la relación posesiva con el objeto, que no puede entenderse fuera de esta lógica.

\section{Imitación y humanidad}

La definición de ambición no puede pensarse al margen de la definición de humanidad, no sólo porque las dos aparecen ligadas en el texto, sino porque comparten prácticamente el mismo mecanismo:

Este esfuerzo de hacer algo y también de omitirlo, con el único objetivo de agradar a los hombres, se llama Ambición <Ambitio $>$, sobre todo cuando ponemos tanto empeño en agradar al vulgo que hacemos u omitimos algo con daño propio o ajeno. En otro caso, suele llamarse Humanidad <Humanitas $>$. ( $E$ III 29 sc.; Spinoza, 2000, p. 146).

La humanidad debe entenderse en paralelo a la ambición y estos dos afectos a su vez dentro del cuadro de la imitación: queremos ser causa del gozo de los otros para reproducir imitativamente el amor que los otros tiene hacia lo que le causa gozo; así, el amor del otro, por la imitación, se convertiría en amor propio. La imitación de los afectos <imitatio affectuum $>$, expuesta en la proposición 27 de la tercera parte de la Ética, se define de la siguiente manera:

Por el solo hecho de imaginar que una cosa, que es semejante a nosotros <res nobis similem $>$ y por la que no hemos sentido afecto alguno <et quam nullo affectu prosecuti sumus $>$, está afectada por algún afecto, somos afectados por un afecto similar. ( $E$ III 27; Spinoza, 2000, p. 144).

La imitación es el gran mecanismo que permite pensar tanto la sociabilidad humana como el conflicto, ligando los dos aspectos: deseamos lo que los otros desean y por ello rivalizamos; deseamos someter a los otros y los otros a nosotros y por ello nos enfrentamos; pero también nos integramos con los demás, tejemos una relación entre los hombres que toma cuerpo en la formación del individuo-humanidad que abarca la totalidad de los hombres.

Es interesante a este respecto recuperar el concepto de Humanidad que Matheron deduce de la exposición de Spinoza. La Humanidad, como Matheron señala, es el conjunto de hombres en su totalidad, unidos por lazos miméticos, que opera como si fuera un único individuo (Matheron, 1969 , p. 155). De esta manera todos los hombres forman el cuerpo de la humanidad. La humanidad, basada en la semejanza, no parte de la concepción imaginaria de cada sujeto. La humanidad no es la proyección de nuestra imagen propia sobre el cuerpo constituido por todos los hombres.

Las razones de esta unión no son voluntarias. Los hombres no deciden voluntariamente unirse a los demás en un individuo común. Voluntariamente deciden distinguirse de los otros creyéndose superiores para así dominarles. La Humanidad se produce en cambio de manera no voluntaria gracias a las propiedades comunes que tienen los hombres, como sostiene Matheron (Matheron, 1969, p. 155). Así, a partir de las propiedades comunes, este individuo tiene su conato propio: los hombres se esfuerzan, unidos, en el bien común a todos. En ese sentido la humanidad se asemeja a la razón, ya que los hombres actúan movidos imitativamente por el bien común, aunque desconozcan el bien común. La imitación no participa de la razón, pero en la medida en la que hace a los hombres colaborar entre ellos, les hace actuar como si fueran guiados por la razón, de la misma manera que el mensaje de Cristo, reducido a la sentencia "ama al prójimo como a ti mismo" ( $G$ III 165; Spinoza, 2014 , p. 230) produce una cierta salvación sin conocimiento que en otra obra Matheron ha llamado "salvación de los ignorantes" (Matheron, 1971, p. 78). Sin embargo, a pesar de señalar este concepto y su origen imitativo, Matheron no explica cómo se produce este tipo de relación. Para explicarlo es necesario, a nuestro juicio, renunciar a una estructura afectiva binaria e introducir un tercer elemento que expondremos a continuación.

Para entender esta relación es necesario comprender de antemano al otro. El otro puede ser concebido de dos maneras: en primer lugar de manera imaginaria, donde el otro, para nosotros, es 
una imagen de nosotros mismos, una proyección, una especie de espejo en el que buscamos ante todo que nuestro amor propio se refleje ${ }^{4}$ : pero en segundo lugar el otro puede ser también concebido como alguien que tiene una naturaleza como la nuestra, una capacidad de sentir y de afectar como la que nosotros tenemos y que nos mueve a perseverar junto con él ${ }^{5}$. ¿Cuál es la opción que predomina? Probablemente las dos al mismo tiempo, ya que, como la descripción espinosista de los afectos interhumanos deja ver, los hombres oscilan de una a la otra. Los dos polos, siempre presentes, son la constante de la vida afectiva caracterizada por el hecho de que ningún polo puede anular al otro, quedando condenados a una especie de equilibrio sin término medio.

Estos polos pueden ser estudiados a partir de las dinámicas que generan. El polo de la imaginación, productor de la rivalidad, genera una dinámica de exclusión (queremos distinguirnos del otro porque es semejante a nosotros) mientras que el polo en el que el otro tiene una consistencia al margen de nuestra imagen genera una dinámica de integración (queremos unirnos al otro porque es semejante a nosotros). La primera dinámica, que es la de la rivalidad y la del amor propio puede, llevada al extremo, como señala Laurent Bove (Bove, 1992, p. 85), conducir a la inhumanidad, es decir a una tensión entre los hombres basada en la primacía egoísta del sujeto imaginario que puede llegar a desear la anulación del otro; la segunda, al contrario, conduce a una concepción de la humanidad como unión de los hombres en un mismo individuo. La humanidad se constituye a partir de una imagen del otro que no proviene de la imagen propia que tenemos de nosotros mismos, lo que hace que el otro no dependa de nosotros para ser algo (como la imagen del espejo depende del modelo), sino que tenga una consistencia al margen de nuestra imagen narcisista.

La clave para entender la humanidad, frente a la lógica de la rivalidad que Spinoza deduce al mismo tiempo de la imitación, reside a nuestro juicio en una comprensión de la mediación. Existen dos tipos de mediación: el primero, que expondremos más adelante, consiste en presentarnos ante el otro como un mediador con el Otro; se trata de una relación de poder en la que nuestro objetivo es someter al otro. El segundo consiste por el contrario en considerar al otro como el mediador entre nosotros y el Otro, entre nosotros y todos los demás, la Humanidad.

En la medida en la que el otro no es una imagen vacía de nosotros mismos sino que se afirma positivamente, el otro se nos aparece como humano, es decir, se nos aparece como miembro de un individuo más amplio que es la humanidad. Esa humanidad, que llamaremos Otro con mayúscula en el sentido en el que, en un principio, está formada por todos los hombres salvo nosotros, por los demás, incluido el otro que tenemos delante. El otro nos pone en contacto con la humanidad, con los Otros, es nuestro mediador. Pero no sólo nos pone en contacto; el otro nos permite al mismo tiempo formar parte de este gran individuo colectivo. Para ello debemos tratarlo humanamente, debemos tratarlo como los hombres se tratan en tanto que humanos, en una lógica que excluye el amor propio en virtud de la cooperación.

La constitución del individuo-Humanidad cambia la lógica de la exclusión por una lógica de la inclusión. La única manera de formar parte del Otro, de la humanidad es con el prójimo. Mostrando nuestro lazo con él en vez de su exclusión del todo nos hacemos partícipes de lo humano, saliendo de la lógica de la dominación. No tratamos al otro como un objeto que poseer, que dominar. Saliendo de esa dialéctica nos integramos en la totalidad, en el individuo común. Ello implica, por supuesto, rechazar nuestra superioridad imaginaria, rechazar la idea de que nosotros somos la cabeza de la comunidad de hombres que vamos a integrar y reconocer que este centro está descentrado, que este Otro no es nadie en concreto.

\section{Dominación}

En paralelo a las relaciones de socialización, cuya estructura terciaria hemos señalado, es necesario estudiar las relaciones de dominación. Distinguiremos dos tipos: en primer lugar, aquellas cuya estructura triangular está compuesta por un objeto en uno de los tres polos; en segundo lugar, aquellas cuyos tres polos están compuesto por figuras humanas o subjetivas. En este segundo lugar distinguiremos dos partes: por un lado un mecanismo que, partiendo del conflicto, tiene como objetivo asegurar la dominación; por otro lado, un mecanismo que, partiendo de la calma social -producida en ciertos casos por la dominación-, reactiva el conflicto para luchar contra la tiranía. 


\section{Rivalidad y propiedad}

Spinoza explica en Ética IV 34 las causas de oposición entre los hombres. Un hombre se opone a otro en primer lugar porque el otro puede asemejarse, según lo expuesto en Ética III $16^{6}$, a algo que odiamos; en segundo lugar porque el otro desea una cosa que deseamos pero que sólo una persona puede poseer, como afirma la proposición 32 de la tercera parte ${ }^{7}$; en tercer lugar por amor propio: los hombres quieren distinguirse de los demás mostrándose superiores a ellos (y para ello una buena manera es poseer lo que sólo uno puede poseer), como explica la proposición 55 de la tercera parte ${ }^{8}$.

En las tres causas de oposición opera una estructura triangular: nuestra relación con A se constituye por la mediación de B. Sin embargo hay una diferencia entre la primera causa y las otras dos. En la primera, odiamos al otro porque se parece a un tercero al que odiamos. La semejanza es el vehículo de las pasiones, tanto del odio como del amor, como se establece en la proposición 16 de la tercera parte que afirma que podemos amar una cosa cuando ésta se parece a algo que amamos y odiarla cuando se parece a algo que odiamos ( $E$ III 16; Spinoza, 2000, p. 138). Este odio no atiende a mecanismos fijos, sino al umbral de la semejanza, que es muy laxo ya que, como señala Alexandre Matheron, "todo se parece un poco a todo" (Matheron, 1969, p. 155). Sin embargo las dos siguientes causas de oposición se articulan en torno a un mecanismo fijo, la rivalidad, que late en el corazón de la propiedad.

En un texto titulado "Spinoza et la propiété" (Matheron, 2011) Matheron elabora, en paralelo a las causas de la oposición que Spinoza enumera en Ética III 34, las bases de la propiedad. Según Matheron el deseo de propiedad se sigue de las tres formas que coinciden con las causas de la oposición. En primer lugar, queremos poseer no sólo los objetos que nos producen gozo, sino aquellos que se parecen a éstos, pues suponemos que, por ser semejantes, nos producirán gozo también. En segundo lugar, si alguien goza de una cosa que sólo uno puede poseer querremos automáticamente arrebatársela para gozar de ella de manera exclusiva. En tercer lugar, queremos poseer ciertas cosas, sobre todo aquellas que los demás estiman, para mostrarnos como superiores, por la posesión, a los otros, que no las poseen.
La primera forma de propiedad que Matheron señala coincide con la primera forma de conflicto: las dos se basan en la semejanza de ciertas cosas o personas con otras cosas o personas que nos resultan agradables o detestables. Sin embargo una novedad se introduce en las dos causas siguientes: no se puede comprender la rivalidad sin la propiedad ni a la inversa. Las dos se implican mutuamente, es decir, las dos comparten la misma estructura triangular. La única diferencia consiste en el orden de los polos.

En la segunda forma de conflicto, la "cosa semejante" es intermediaria entre nosotros y lo que deseamos poseer. En cambio, en la tercera forma, el objeto que queremos poseer es intermediario entre nosotros y nuestro rival. Al imaginar una cosa que sólo uno puede poseer, afirma Spinoza, queremos gozar exclusivamente de ella, al igual que los otros, quienes devienen por ello automáticamente nuestros rivales ( $E$ III 32; Spinoza, 2000, p. 148). Sin embargo, si queremos poseer dicha cosa no es simplemente por el gozo que puede aportarnos, sino por el hecho de aumentar nuestro amor propio al considerarnos superiores a los demás (y, sobre todo, siendo reconocidos por ellos como tal) que no son capaces de poseerla. Así, una cosa implica la otra: los otros median entre nosotros y lo deseado, en la medida en la que gran parte de las cosas que deseamos provienen de la emulación ( $E$ III 27 sc.; Spinoza, 2000, p. 144), que es el origen de la rivalidad ante un objeto'; pero, por otro lado, el objeto deseado deviene una mediación en el momento en el que a través de él queremos cumplir nuestro verdadero deseo, que es ser superior a los demás.

Se configura así una estructura afectiva triangular: nuestra relación con los objetos pasa por el otro (rivalidad), a la vez que nuestra relación con los otros pasa por las cosas (propiedad). Es así como propiedad y rivalidad forman una estructura afectiva clave para el desarrollo de las relaciones humanas, completamente atravesadas por lo imaginario. Necesitamos a los demás para representarnos, por la emulación, cosas que sólo uno puede poseer pero a su vez necesitamos a los demás para imaginarnos superiores a ellos. Así, en esta relación al objeto, el otro deviene mediador frente a nosotros mismos, entre la imagen que tenemos (lo que somos) y la que nos gustaría tener (ser superiores al resto). Esta estructura afectiva se introduce en el seno de toda teoría imaginaria, 
pues lo que mueve nuestras acciones, desde un punto de vista imaginario, es esta dialéctica de posesión-rivalidad, que consiste en "poseer" a los otros, hacerles reconocedores de nuestra grandeza, poseyendo lo que desean.

Nuestra relación a nosotros mismos pasa por los otros dos polos del triángulo. Por un lado imitamos al otro, viviendo a su manera y deseando lo que desea, y por otro nos sometemos a él, volviéndonos una simple apariencia, volviéndonos una imagen (ser-para-el-otro) que el otro reconoce como superior pero que no es más que una imagen.

\section{La dominación como causa conflicto}

Los hombres, para sentirse superiores a los otros y hacer que estos se sometan, buscan poseer algo que todos deseen pero que al mismo tiempo nadie posea: sólo así creen que serán reconocidos como superiores por los otros. Sin embargo, la posesión de un objeto semejante no calmará el conflicto sino que lo aumentará, ya que los otros estarán envidiosos e intentarán de arrebatarle el objeto que le otorga la superioridad. Así, nadie se someterá a quien posee lo que todos desean, sino que todos, en la medida en la que se creen superiores al resto, se creerán legítimos poseedores de lo que solo uno puede gozar y rivalizarán para obtenerlo. ¿Es posible que un hombre sea considerado como superior por los demás sin despertar la envidia, la rivalidad y la amenaza? La respuesta parece ser no.

Es probablemente en el Tratado Teológico Político donde mejor se ilustra esta ambición tiránica por dos razones: en primer lugar por el hecho de que en este tratado, a diferencia de la Ética o del Tratado Político, Spinoza no ha desarrollado la contradicción que hace que los hombres hagan lo que los demás desean, estableciendo las bases de lo social. Sin embargo, esta situación no es exclusiva de este tratado porque, como claramente lo señala Matheron, la ambición de gloria que teje los lazos entre los hombres nunca es capaz de eliminar completamente la ambición de dominación que, en un momento o en otro, se acaba imponiendo, al mantenerse el deseo de imponernos a los otros (Matheron, 1969, p. 1968). En segundo lugar, porque en es en este tratado donde más claramente Spinoza critica la tiranía y la dominación despótica, aunque en sea en sus obras posteriores donde introduzca la categoría de indignación, esencial para comprender la tensión producida por el despotismo.

A continuación pasaremos a analizar esta estructura de la dominación, que es triangular, a partir de los mecanismos expuestos tanto por Spinoza como por sus comentadores. Distinguiremos dos grupos: por un lado los afectos o mecanismos que partiendo de la lucha por la dominación son capaces de instaurar un orden sociopolítico; por otro lado los afectos o mecanismos que, dentro de un orden sociopolítico vigente, son capaces de disgregarlo. Hay que distinguir en este último punto dos tipos de estructuras triangulares. La primera es capaz, después de la disolución, de proponer las bases de un nuevo sistema; la segunda en cambio disuelve el sistema sociopolítico sin capacidad para imponer uno nuevo.

\section{La indignación}

El mecanismo de la indignación, presentado por Spinoza en Ética III 22 escolio y definición de los afectos. Se define de este modo: "La indignación es el odio hacia alguien que hizo mal a otro" (III 22; Spinoza, 2000, p. 142). La indignación es uno de los pocos mecanismos que Spinoza reconoce explícitamente como constituyentes de una estructura triangular formada completamente por personas y no por un objeto. En la medida en la que alguien causa tristeza a un semejante nos produce odio. Odiamos a los que hacen mal a nuestros semejantes, los que le afectan de tristeza. Como señala la explicación de la definición de indignación, este mecanismo reenvía al mecanismo imitativo. La imitación afectiva nos hace desear lo que otros desean y sufrir con las tristezas de los demás. El odio es el afecto que se produce cuando una cosa disminuye nuestra capacidad de actuar, esto es, nos causa tristeza. Cuando vemos a otro triste compartimos su tristeza, pero también su odio hacia lo que le entristece. En este sentido, imitación e indignación parecen ser las dos caras de un mismo mecanismo. La indignación aparece cuando el tercero, el tercer elemento del triángulo afectivo, no es una cosa sino una persona. En ese sentido nos ligamos a la víctima en una relación de odio frente al Otro. Lo que nos une es nuestra humanidad: no soportamos que un tercero haga algo insoportable al prójimo.

En este sentido, la indignación es una de las causas principales de la disolución del Estado ( $G$ III 292; Spinoza, 1986, p. 115). En el momento 
en el que el soberano entristece de manera injustificada al prójimo, la multitud, por empatía con el prójimo, se une frente al soberano. Los súbditos se separan de él, deshaciendo el cuerpo del Estado, dejándolo como una simple arquitectura ilusoria. El soberano quiere hacer permanecer la estructura, el cuerpo del Estado, pero los ciudadanos ya no pertenecen a él, están fuera, son el otro-excluso.

Gracias a la indignación, los hombres se unen entre sí, se unen con al otro frente un tercero que hace daño a los semejantes. Así, la indignación funciona, como señala Matheron, por el linchamiento (Matheron, 1994, p. 164), o dicho de otro modo, por el chivo expiatorio. Esto, que en un primer momento implica la disolución del Estado, al introducir la división en el seno de la sociedad, implica, a su vez, la restructuración en un nuevo cuerpo político e implica una nueva unión entre los hombres. Aunque al producir tristeza y odio, debe ser considerada como una afección mala, la indignación puede, por ser una forma de imitación afectiva, unir a los hombres, construyendo afectos comunes y haciendo que actúen como si estos fueran una sola mente, o un solo cuerpo.

\section{Identificación}

En el parágrafo 4 del capítulo X del Tratado Político, Spinoza explica como ciertos hombres, quienes una vez perdido el miedo al terminar la guerra se han vuelto flojos y cobardes, se apropian de las costumbres de los extranjeros para mostrarse superiores a los prójimos:

\footnotetext{
No podrá, sin embargo, evitar que se infiltren los vicios que no pueden ser prohibidos por una ley, como son aquellos en que caen los hombres que gozan de tiempo libre y de los cuales no rara vez se sigue la ruina del Estado. Porque los hombres en la paz, tan pronto deponen el miedo, se transforman paulatinamente de feroces y bárbaros en civilizados o humanos, y de humanos en blandengues e inactivos. Lejos de emularse unos a otros en la virtud, se emulan en la fastuosidad y en el lujo. Pronto comienzan, pues, a sentir hastío de las costumbres patrias y a adoptar las ajenas, es decir, a ser esclavos. ( $G$ III 350; Spinoza, 1986, p. 215).
}

Spinoza muestra explícitamente el mecanismo triangular del deseo a partir de este mecanismo que en otro trabajo hemos propuesto llamar identificación ${ }^{10}$. Para mostrarse como superiores a los prochains y así dominarlos, ciertos hombres se presentan como partícipes de las costumbres de los extranjeros. Así, estos hombres aparecen frente a los demás no sólo como extravagantes, al hacer cosas que los demás no hacen, sino superiores, como modelo de los extranjeros. Con ello se muestran como si fueran parte de los extranjeros, movilizando a esta figura del extranjero, lejana e incluso inaccesible, frente al prójimo al que quieren someter. Ante el extranjero, que aparece como Otro, el prójimo, admirativo, debe -se supone- reconocer por un lado la superioridad de las costumbres ajenas, no por ser superiores en realidad, sino por nunca vistas, y por otro lado intentar ganarse los afectos de los extravagantes en la medida en la que parece que controlan al Otro-extranjero (que, suponemos por el texto, pudo ser anteriormente el enemigo).

Tomar las costumbres del Otro hace decaer las propias, introduce la envidia y activa la rivalidad. Así, el cuerpo de la sociedad deja de comportarse como uno, y las partes comienzan a intentar diferenciarse entre ellas, produciendo el conflicto. "Dado que los hombres se guían, como hemos dicho, más por la pasión que por la razón, la multitud tiende naturalmente a asociarse, no porque la guíe la razón, sino algún sentimiento común" ( $G$ III 297; Spinoza, 1986, p. 112); si este sentimiento común, esta unidad, se disuelve, se entra en un estado de conflicto.

Este conflicto conlleva a su vez una doble alienación: la primera, ya mencionada, al convertirse en una imagen para el otro (ser-para-el-otro) en la medida que, para que el prójimo nos vea como superiores dejamos de ser lo que somos para volvernos lo que él querría ser; la segunda, la pérdida de nuestras costumbres propias, nuestra forma de ser, nuestro ingenio en definitiva, y adopción del ingenio del Otro ${ }^{11}$. Así se produce una situación más compleja que la anunciada en el concepto de ambición: para gustar al otro necesitamos ser lo que el otro quiere ser, esto es, el Otro, la totalidad, y para ello tenemos que alinearnos doblemente.

Frente a la alienación de la imitaciónambición, capaz de producir un tejido afectivo de socialización primaria, esta nueva estructura, al ser triangular por un lado y, por el otro, implicar dos formas paralelas de alienación, no puede resolverse como la anterior en una forma de socialización mínima. Por el contrario, no hace sino deshacer 
toda forma de sociabilidad al acentuar la fractura imaginaria. La manera por la que los hombres buscan gustar a los demás esconde una forma de dominación que destruye el equilibrio social. Por ello, la identificación lleva a la disolución de la sociedad.

\section{La dominación como fin del conflicto}

Para contrarrestar esta tiranía que todos los hombres quieren imponer es necesario crear, al menos de manera ilusoria, las condiciones de la sumisión del otro por una lógica de la exclusión a través del premier tipo de mediación anteriormente señalado. El individuo que quiere dominar a otro intentará mostrarle que el resto, los demás, ya están bajo su poder. De esta manera, el otro se verá acorralado y no tendrá más remedio, para no ser excluido de la totalidad, que integrarse. Hadi Rizk encuentra un mecanismo en el Tratado Teológico-político para hacer frente al conflicto. Este consiste, para Rizk, en tejer un lazo entre yo (dominador) y los Otros, <le reste $>$, los demás, la totalidad, presentando a la víctima (el otro) como el único elemento exterior al todo:

El acuerdo patológicamente extorsionado no es finalmente más que la representación ilusoria, ficticia, de la unificación de la multitud por los solos recursos de la dinámica de un tercero, quien junta bajo la figura del Otro absoluto el ser-Otro de todos las relaciones inadecuadas. Esta "mediación" produce una unión inmanente a la relación humana, aunque bajo las especies de la más pobre síntesis, esto es de una impotencia común hacia el poder colectivo. (Rizk, 1995, p. 116).

La figura del Otro como potencia descomunal inclina la balanza. El otro, el prójimo, si no quiere oponerse al Otro, que está de nuestra parte, tiene que venirse a nuestro grupo. En este sentido se pone a nuestra disposición para no ser excluido de la totalidad (formada de manera ilusoria por el Otro más nosotros). Por ello el juego de la dominación consiste en hacer creer al otro que existe un Otro y que está de nuestra parte, que este Otro nos obedece o que formamos un todo con él. "Ningún $\mathrm{S}$ puede dominar A si no hace creer a este último que $\mathrm{B}$ o $\mathrm{C}$ ya están en su poder y al mismo tiempo lo aumentan" (Rizk, 1995, p. 116). Esta lógica moviliza otra forma de alteridad: yo y los demás somos uno; ellos me pertenecen, están bajo mi poder y lo refuerzan. Así, si tú no te sometes a mí, si no te unes a nosotros, estarás fuera del grupo, serás nuestro potencial enemigo e iremos, en caso de necesidad, contra ti. Se produce así, señala Rizk, de manera ilusoria,

la unificación, contra tal individuo, de los demás. Estos últimos son así tomados de manera colectiva en razón de su síntesis pasiva en un resto y este resto se confunde con la unión, menos tal individuo, de los demás. Él está unido al resto, constituido por la serie como su recíproco desigual, en una relación de alteridad, de dominación real y de exclusión potencial. (Rizk, 1995, p. 116).

Así, el dominador tiene acceso al Otro, es capaz de movilizar, de manera ilusoria, el poder de la multitud, contra aquel a quien quiere someter. ¿Quién es el Otro? Una ilusión creada que representa a la totalidad frente al otro, una totalidad que excluye al otro y de la que el otro no puede sino querer formar parte.

Según Rizk, el gobernante es, en última instancia, el que aparece ante los súbditos como aquel que tiene la capacidad de desplegar todo el poder de la multitud, del cuerpo político, contra cada súbito particular. Este mecanismo de constitución de lo político referido según Rizk a Moisés, paradigma del gobernante en el Tratado Teológico-Político, introduce de nuevo la problemática de la mediación. Si en el caso anterior la mediación se constituía entre objetos y sujetos, en una dialéctica de sujeto, el mecanismo mediador constitutivo de lo político implica otro tipo de mediación. El dominador se presenta como mediación entre el dominado y lo Otro, como aquel que tiene el poder de la multitud en su totalidad, como es el caso de Moisés, pero también el poder de la divinidad. Moisés de esta manera controla a Dios, en el sentido en el que no es él el que está de parte de Dios sino que, a ojos del pueblo, es Dios quien está de su parte. Esta forma de mediación introduce estructura teológica en la constitución de lo social. Hay una equiparación entre el Otro, la potencia de la totalidad menos uno, menos el aun no dominado, y Dios, la totalidad de las fuerzas del cosmos. La estructura teológica aparece como una forma de sumisión, como uno de los mecanismos de sumisión empleados por la ilusión y el engaño para ligar a los hombres a un cuerpo político que, a pesar de ser ilusorio en su comienzo, se vuelve 
real por la participación común. Los hombres, por lazos inicialmente imaginarios, someten al mediador formando una unión real que ya no sólo se basa en una serie de imágenes, sino en la incorporación, en la constitución de un cuerpo político que funciona.

La tesis de Rizk, a pesar de su originalidad, implica algunos problemas. Por un lado, al basarse en la figura de Moisés, esta tesis remite claramente Tratado Teológico Político; sin embargo en este tratado Spinoza expone de manera explícita la formación de lo social en términos contractualitas, lo que invalidaría la tesis de Rizk ${ }^{12}$. En efecto, si la sociedad proviene de un pacto, como señala en el capítulo dieciséis de dicho tratado, y este pacto no es sino el fruto del común acuerdo, el mecanismo que Moisés representa quedaría invalidado. Por otro lado, aplicar esta tesis al Tratado Político para explicar la formación de lo social resulta superfluo, ya que no hay paso del estado de naturaleza al estado civil gracias a una capa de sociabilidad primaria construida mecanismos como la imitación o afectos como la ambición ${ }^{13}$. Por ello, a nuestro juicio, este mecanismo no debe comprenderse como generador de lo político sino como un mecanismo que, como la indignación aunque desde una perspectiva contraria- es capaz de reconstituir un cuerpo social que se encuentra quebrado por el conflicto.

\section{Conclusión: la producción de deseo.}

Las principales relaciones afectivas en la obra de Spinoza no son binarias, sujeto-objeto, sino terciarias. Una concepción triangular de las relaciones afectivas permite mostrar la articulación de ciertas dinámicas interhumanas basadas en la figura de un tercero que, aunque no siempre aparezca de manera evidente, siempre está presente. La ventaja de esta lectura es doble; permite tanto explicar la formación de las relaciones afectivas como la configuración de las formas subjetivas.

Hay una conexión que debe ser señalada a modo de conclusión entre la estructura triangular de los afectos y el deseo que circula entre los polos que la constituyen. A pesar de que el deseo, que Spinoza asimila a la esencia del hombre, no es más que un esfuerzo por perseverar en el ser, su comprensión en abstracto resulta extremadamente pobre. La mayor parte de las formas por las que un hombre persevera no se explican solamente por la capacidad individual de producir o reproducir representaciones sino que provienen de los demás.

En este sentido la noción de mediación anteriormente señalada es clave. En la estructura triangular uno de los polos siempre juega el rol de mediar ante los demás. Este rol mediador, imaginario la mayor parte de las veces, permite una reformulación o elaboración del orden de la estructura, siempre triangular, y permite comprender los cambios de circulación del deseo en el marco de las relaciones interhumanas. El ejemplo de la propiedad es tal vez el más claro: el objeto puede ser mediador entre dos personas (que por lo tanto rivalizan para conseguirlo), pero también es posible que el objeto sea mediado por una persona (lo que llevaría a la envidia o a los celos) ( $E$ III 32 sc.; Spinoza, 2000, p. 150). En los dos casos, la noción de objeto que está en juego, como Spinoza apunta, proviene de una concepción imaginaria que concibe al objeto como exclusivo, esto es, como si sólo pudiera ser poseído por una persona ( $E$ III 32; Spinoza, 2000, p. 148). En este sentido el deseo se produce con y por la mediación: deseamos lo que otro posee, de manera imitativa; pero también deseamos algo para sobrepasar al otro, para mostrar nuestra superioridad.

Es también la mediación lo que explica el deseo de dominar a los demás, el hecho de intentar que el otro se someta a nuestro deseo, que persevere a nuestra manera, que se convierta de algún modo en un complemento para nuestra conservación. Pero la mediación juega a su vez un rol importante en la producción de otro tipo de deseo, que es el deseo como conservación común de un grupo de individuos, esto es, el llamado individuo-Humanidad. Se trata de un deseo común a todos los hombres que, por el hecho de ser común, no puede basarse en representaciones singulares y exclusivas: una perseverancia en común implica un deseo común cuyo objeto tiene que ser compartido necesariamente por todos los que juntos perseveran. Se trata por ello de un objeto que no solamente tiene la capacidad de poder ser compartido sino que además sólo puede obtenerse de manera compartida, en común, lo que hace que los hombres tengan que unirse para su consecución. Esta producción de deseo está muy ligada a la producción de nociones comunes con ello a la producción del gozo de la racionalidad.

Es por todo esto que la circulación triangular es parte esencial de no sólo de las relaciones entre 
sujeto-objeto, sino del paso del assujetissement, es decir de la sumisión concebida como incorporación del otro a un deseo exclusivo de nosotros mismos, a la formación de un sujeto colectivo que persevera de manera colectiva y en cuyo esfuerzo común se dibuja la constitución gozosa de la razón. 


\section{Referencias}

Bove, L. (1992). La stratégie du conatus. Paris: Vrin.

Gracián, B. (2001). El héroe. Barcelona: Estrategia Local, S. A. Recuperado de http://www.estrategialocal.cat/_ca/libros_y_manuales/_internal/repository/HEROE.pdf

La Rochefoucauld. (1971). Maximes. Paris: GF Flammarion.

Lazzeri, C. (1998). Droit, pouvoir et liberté. Spinoza critique de Hobbes. Paris: PUF.

Macherey, P. (1995). Introduction à l'Éthique de Spinoza. Troisième partie, la vie affective. Paris: PUF.

Matheron, A. (1969). Individu et communauté chez Spinoza. Paris: Éditions de minuit.

Matheron, A. (1971). Le Christ et le salut des ignorants. Paris: Aubier-Montaigne.

Matheron, A. (1994). L'indignation et le conatus de l'État spinoziste. En M. Revault d'Allonnes \& H. Rizk (Eds.), Spinoza. Puissance et ontologie. Paris: Kimé.
Matheron, A. (2011). Spinoza et la propriété. En A. Matheron Études sur Spinoza et les philosophies de l'âge classique. Lyon: ENS éd.

Moreau, P.-F. (2009). El concepto de ingenium en la obra de Spinoza. Ingenium. Revista de historia del pensamiento moderno, 1, 3-12.

Soulier, P. (2000). Pour en finir avec le contrat social. Autres Temps, Cahiers d'éthique sociale et politique, 68, 59-73.

Spinoza, B. (1986). Tratado Político. Madrid: Alianza Editorial. Spinoza, B. (2000). Ética. Madrid: Trotta.

Spinoza, B. (2014). Tratado Teológico-político. Madrid: Alianza.

Rizk, H. (1995). Les affects du pouvoir. Rue Descartes, 12-13 (Passions et politique), 106-121. 
1 "El conato con el que cada cosa se esfuerza en perseverar en su ser, no es nada más que la esencia actual de la misma" ( $E$ III 7; Spinoza, 2000, p. 133).

2 "Tanto si tiene ideas claras y distintas como si las tiene confusas, el alma se esfuerza en perseverar en su ser por una duración indefinida, y tiene conciencia de ese esfuerzo suyo" ( $E$ III 9; Spinoza, 2000, p. 133).

Este conato, cuando se refiere sólo al alma, se llama voluntad; en cambio, cuando se refiere a la vez al alma y al cuerpo, se llama apetito. Este no es, pues, otra cosa que la misma esencia del hombre, de cuya naturaleza se sigue necesariamente aquello que contribuye a su conservación y que el hombre está, por tanto, determinado a realizar. Por lo demás, entre apetito y deseo no hay diferencia alguna, excepto que el deseo suele atribuirse a los hombres en cuanto que son conscientes de su apetito; y por tanto puede definirse así: el deseo es el apetito con la conciencia del mismo. Por todo esto consta, pues, que nosotros no nos esforzamos, queremos, apetecemos ni deseamos algo porque juzgamos que es bueno, sino que, por el contrario, juzgamos que algo es bueno, porque nos esforzamos por ello, lo queremos, apetecemos y deseamos. $(E$ III 9 sc.; Spinoza, 2000, p. 134).

Spinoza identifica el conato y el apetito, y distingue el apetito del deseo por la ausencia de conciencia en el primero. Sin embargo, si aceptamos por un lado el paralelismo y lo que de él se sigue ( $E$ II 23), y si por otro lado, como se establece en la proposición citada, afirmamos el alma tiene siempre conciencia de su esfuerzo, ese esfuerzo será siempre deseo.

$4 \quad$ Esta la concepción de Andrea Zainetti:

Dans les relations intersubjectives, le mécanisme de projection est inévitablement destructeur, parce que chaque personne s'efforce, autant qu'elle peut, d'aimer dans l'autre la totalité d'elle-même et d'haïr ce qui la nie en partie ou en totalité. Comme l'autre ne convient que partiellement au rapport qui définit notre essence, et qu'il n'entend pas être nié dans les parties qui ne nous conviennent pas chez lui (...) cet autre va nécessairement nous haïr pour l'avoir nié et s'efforcera donc de nous détruire, en partie du moins. Ainsi et c'est le point capital, la destruction de notre essence est nécessairement provoquée par nous-mêmes, puisque nous voulons forcer les autres à vivre selon notre propre naturel. (Zainetti, 2001, p. 104).

De Pierre Macherey: "Este otro que imaginamos, y cuya idea nos persigue obsesivamente, no es más que una proyección mimética de nosotros mismos que nos sigue como una sombra o nos planta cara como una imagen especular, privada de existencia autónoma" (Macherey, 1995, p. 223) y también, aunque de manera menos clara de Pierre-François Moreau :
Los hombres, incluso cuando poseen un ingenium más rico, juzgan necesariamente del ingenium del prójimo a partir del suyo: en efecto, interpretan sus propias acciones en función de un esquema finalista que expresa su ingenium y no el del prójimo, como tampoco el de la realidad; por tanto, cuando intentan descifrar el comportamiento de los demás, si carecen de otros medios para conocerlo, les aplican este esquema. (Moreau, 2009, p. 6).

5 Esta es la concepción de Alexandre Matheron : "L' « imitation des sentiments d'autrui; imitation due elle-même à la ressemblance d'autrui avec nous, c'est-à-dire (le contexte est explicite) à la propriété qu'il partage avec nous d'être homme" (1969, p. 154).

Por el solo hecho de que imaginamos que una cosa tiene algo semejante a un objeto que suele afectar al alma de alegría o tristeza, aunque aquello en que la cosa es semejante al objeto no sea la causa eficiente de estos afectos, la amaremos o la odiaremos. (Spinoza, 2000, p. 138).

7 "Si imaginamos que alguien goza de una cosa que uno solo puede poseer, nos esforzaremos en lograr que no la posea". (Spinoza, 2000, p. 148).

Por el solo hecho de que el alma imagina su impotencia, se entristece ( $E$ III 55). Pues siempre que uno imagina las propias acciones, es afectado (por 3/53) de alegría, y tanto mayor cuanta más perfección imagina que expresan sus acciones y más distintamente las imagina, esto es (por lo dicho en $2 / 40 \mathrm{el}$ ), cuanto más puede distinguirlas de las demás y contemplarlas como cosas singulares. De ahí que cada uno gozará al máximo con su propia contemplación, cuando contempla en sí algo que niega de los demás. En cambio, si lo que afirma de sí mismo, lo refiere a la idea universal de hombre o de animal, no gozará tanto; y, por el contrario, se entristecerá, si imagina que sus acciones, comparadas a las de los demás, son más débiles; y se esforzará por alejar esta tristeza (por 3/28), ya sea interpretando torcidamente las acciones de sus iguales, ya sea adornando cuanto pueda las suyas. ( $E$ III 55 sc. 1; Spinoza, 2000, p. 163).

$9 \quad$ Pierre Macherey propone incluso de traducir el término latino aemulatio por rivalité en francés (Macherey, 1995, p. 221).

10 Donoso Gómez, Mario "Imitación e identificación en la filosofía de Spinoza" en Revista de filosofía, Madrid (en cola para edición).

11 Esta problemática, que no ha sido trabajada por Spinoza más allá del texto señalado, ha sido sin embargo desarrollada 
por otros autores de su época como Gracián (2001, p. 55) o La Rochefoucauld (1977, p. 131)

Veremos con toda claridad que, para vivir seguros y lo mejor posible, los hombres tuvieron que unir necesariamente sus esfuerzos. Hicieron, pues, que el derecho a todas las cosas, que cada uno tenía por naturaleza, lo poseyeran todos colectivamente y que en adelante ya no estuviera determinado según la fuerza y el apetito de cada individuo, sino según el poder y la voluntad de todos a la vez. (...) Por eso debieron establecer, con la máxima firmeza y mediante un pacto, dirigirlo todo por el solo dictamen de la razón (al que nadie se atreve a oponerse abiertamente por no ser tenido por loco) y frenar el apetito en cuanto aconseje algo en perjuicio de otro, no hacer a nadie lo que no se quiere que le hagan a uno, y defender, finalmente, el derecho ajeno como el suyo propio. ( $G$ III 191; Spinoza, 2014, p. 320).

13 Numerosos autores afirman que en efecto el pacto social expuesto en el Tratado Teológico Político es superado por la sociabilidad conflictual que se introduce con la imitación afectiva. Véase la comparación entre Hobbes y Spinoza (Lazzeri, 1998, pp. 77-93). Véase igualmente Soulier 2000, p. 62 . 